
EDUCATION

Karl Siebold, Embry-Riddle Aeronautical University-Prescott

Phillip Anz-Meador, Embry-Riddle Aeronautical University-Prescott

Ron Madler, Embry-Riddle Aeronautical University-Prescott 


\title{
Space Debris Awareness in Aerospace Engineering Education
}

\begin{abstract}
The authors have taught many space related classes for different universities and distance learning programs at the NASA Johnson Space Center and at Embry Riddle's Extended Campus (Houston) and its residential campus in Prescott, Arizona. The student clientele were aerospace professionals in Houston or full-time undergraduate students in Prescott. The topic of space debris was introduced in these classes and it was found that this field can serve as a very elaborate example pool for applied orbital mechanics, mission planning, spacecraft design (bus and payload), remote sensing and space surveillance, and classes in a traditional liberal arts curriculum such as history, policy, and law.

Projects like the analysis of satellite fragmentations, interactive web based flux directionality calculations, and the long term effects of perturbations on a satellite's orbit are a few examples on how this important topic can be included in a university curriculum. Undergraduate students have been integrated into research projects in addition to the classes offered in a typical curriculum, with benefit both to students and faculty. Additionally, a university can serve as a repository of knowledge, which extends beyond the life of an industrial contractual relationship.

This paper presents methods on how to implement into an aerospace engineering or space physics curriculum the knowledge and foundation needed to understand space debris problems, and by extension many interdisciplinary problems and other areas of science and technology.
\end{abstract}

\section{Astronautics Courses \& Space Debris Education}

The topic of space debris affects three of ERAU's major programs in Prescott, AZ. These programs are Aerospace Engineering, Space Physics, and Global Security \& Intelligence Studies. The interdisciplinary nature of space debris problems becomes evident if one assesses the level of understanding a student from the different programs can have and the contributions a student can make to solving various problems. By nature, a physics and engineering student share the same talents and expertise. However, there may be cases where the sensor requirements levied by the science payload (physics) poses an impossible engineering task. Engineers may design a spacecraft bus which yields the science payload unusable. Writing a well understood space policy requires a high level of understanding of the physics and engineering challenges. By training and experience, the authors possess several decades of relevant experience in the field of orbital debris and space environment studies. Rather than introduce students to orbital debris in a single, probably elective, course, our strategy has been to utilize orbital debris studies as one of several themes within the existing curriculum. The following table (Table 1.1) lists courses and topics where the authors have implemented space debris relevant materials in the curriculum. Section 3 describes a few examples which have been successfully adapted in a classroom setting. The importance in the following list of courses lies in the multitude of opportunities in which space debris problems can be presented. Table 1.1 shows also a list of space debris topics and their relationship with major courses taught, in one form or another, at any university or college supporting a space engineering or physics program. In Table 1.1 our 
course level is indicated, as appropriate, by degree plan year, e.g. " 3 " indicates a Junior-level class.

The intention of this paper is to stimulate the usage of problem solving techniques which can raise the awareness of space debris and give interesting, realistic examples for the classroom. Section 3 contains a non-exhaustive list of examples which illustrate that the field of space debris is very complex and interdisciplinary requiring, as it does, an integrated understanding of dynamics, mathematics and statistics, design best practices, the space environment, and even space history and policy. Once a certain target topic has been set it is a relatively straight forward process in teaching the necessary tools for understanding the problem. However as the following examples will show, it requires some planning and synthesis of previously learned materials.
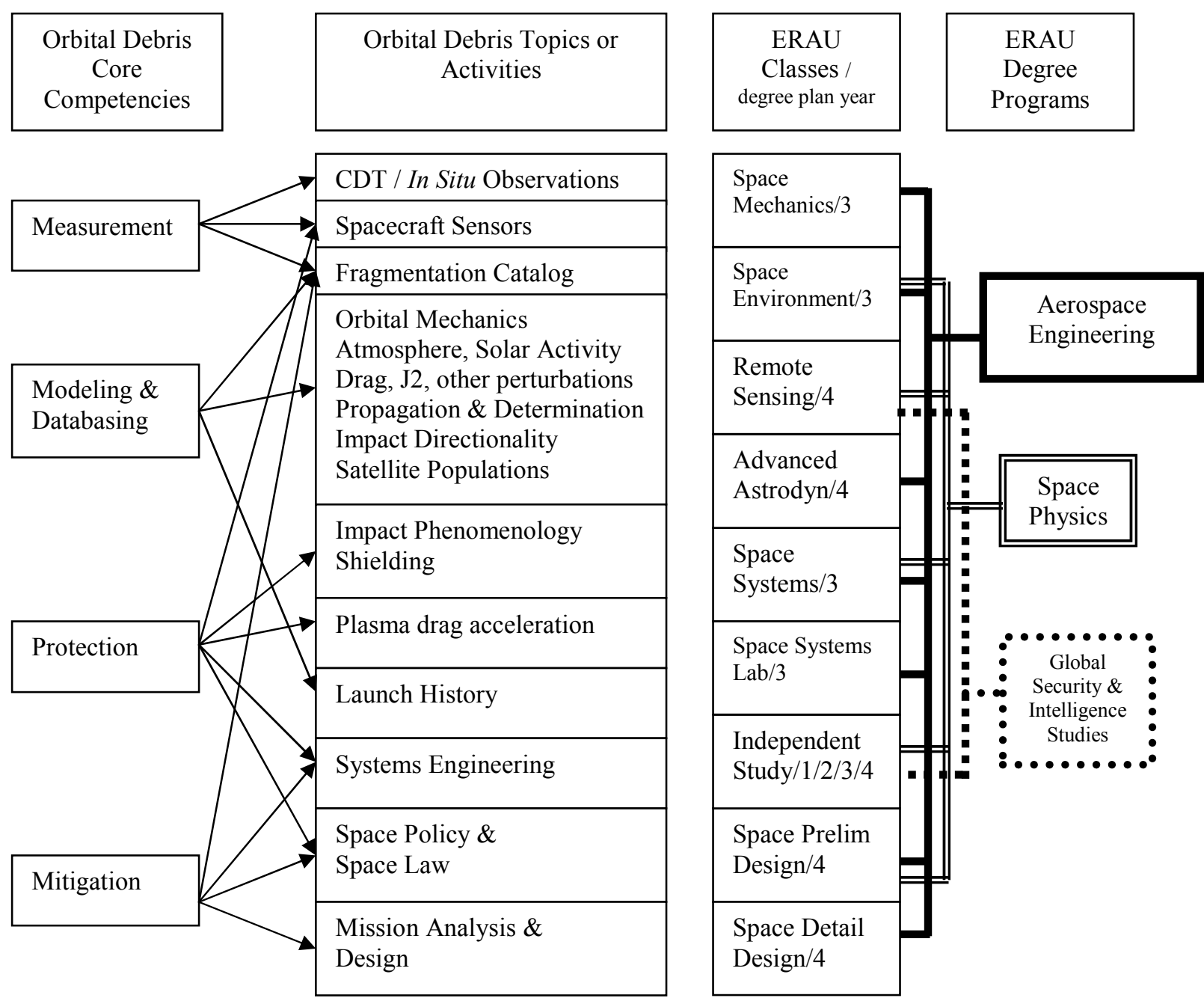

Table 1.1

Orbital Debris Core competencies are those utilized by NASA and the international Inter-Agency Debris Coordination (IADC) committee. Topics and activities are discussed at length in Section 2 of this paper. ERAU classes and degree programs are linked to demonstrate the interaction of students in disparate degree programs in the ERAU Prescott Colleges of Engineering (CoE) and Arts \& Sciences (CoAS). 


\section{Space Debris Course Work and Projects}

The following examples have been used in different Junior/Senior level courses, where the prerequisites are the typical engineering math, physics, statics, and dynamics classes. Even though the following examples could be used to create a single "Space Debris" class, which would be an elective, it is beneficial to spread out the material into different space related classes in order to expose a higher student population to this topic.

\section{$2.1 \quad$ Flux Directionality Example}

One example for the usage of a space debris topic is the calculation of flux directionality of a debris cloud on a spacecraft. This derivation is not as elaborate as Kessler's [1], but it proves the point of the direction of the incoming flux. The following is a classroom example for typical space engineering and space physics related courses. The following derivation can be done in a one hour class, which could also include the MATLAB programming of this example. The following notes give a guideline:

The assumption that the debris cloud with an inclination $i_{2}=100^{\circ}$ and the space craft with an inclination $\mathrm{i}_{1}=50^{\circ}$ are in circular orbits with a velocity $\mathrm{V}$, yields a relationship between the impact angle $\Phi$ and the angle between the orbital planes $\Delta \mathrm{i}$ (Figure 2.1). Due to the $\mathbf{J}_{\mathbf{2}}$ perturbations caused by the Earth's oblateness we can reasonably assume that the distribution of the difference in the right ascension of the ascending nodes $\Delta \Omega$ is uniform. From cosine law in spherical trigonometry follows:

$\cos \Delta i=\cos i_{1} \cos i_{2}+\sin i_{1} \sin i_{2} \cos \Delta \Omega$

solving for $\Delta \Omega$ :

$$
\Delta \Omega=a \cos \frac{\cos \Delta i-\cos i_{1} \cos i_{2}}{\sin i_{1} \sin i_{2}}
$$

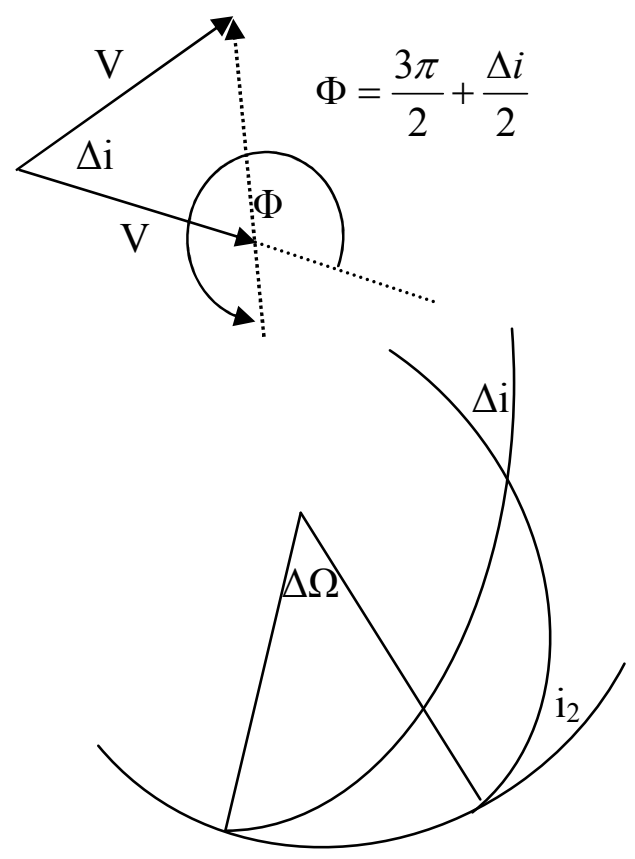

Figure 2.1

The probability density function for the right ascension difference is uniform. Therefore for the probability density of the impact angle $\Phi$ can be deducted as follows:

$$
\begin{aligned}
& \frac{d P}{d \Phi}=\frac{d P}{d \Omega} \frac{d \Omega}{d \Delta i} \frac{d \Delta i}{d \Phi} \text { (2.3) } \frac{d P}{d \Omega}=\frac{1}{2 \pi} \\
& \frac{d \Omega}{d \Delta i}=\frac{1}{\sqrt{1-\left(\frac{\cos \Delta i-\cos i_{1} \cos i_{2}}{\sin i_{1} \sin i_{2}}\right)^{2}} \frac{\sin \Delta i}{\sin i_{1} \sin i_{2}}}
\end{aligned}
$$


It is obvious that not each $\Phi$ will map into a real probability density and the following polar plot can be drawn (Figure 2.2). The result yields the direction of possible debris impacts from a given debris cloud, if its right ascension of the ascending node is uniformly distributed.

Furthermore the keywords printed in bold above, give an idea about the topics to be covered in previous lectures or courses. This example illustrates also the interdisciplinary nature of most space debris related problems. Additionally the MATLAB programming language has been successfully used during a classroom setting for plotting the results in a graph. The code, which is generated by the students in a homework assignment is surprisingly simple, considering the results it delivers. The above example was very well received in graduate level physics and space engineering classes at the Universities of Houston/Clear Lake and Colorado/Colorado Springs where the

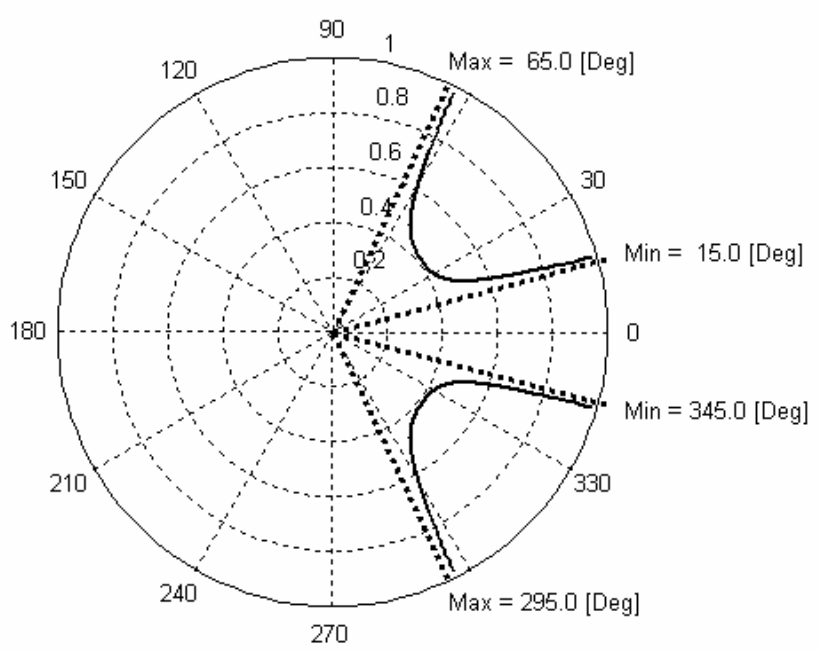

Figure 2.2 student clientele was mostly NASA/JSC Mission Operations employees. It also received a very positive reception in undergraduate classes at Embry Riddle Aeronautical University in Prescott Arizona.

\section{$2.2 \quad$ Asteroid Example}

Another very interesting topic, which sparks a lot of interest from students, is the orbit determination of an asteroid [3] and the calculation of impact possibility with the Earth. In reality such a discovery is made by a set of observations made during a fairly short time interval (Figure 2.3). The first observation of object K02E06Lwas made in 2002, March 10.13090 with a right ascension of $14 \mathrm{hrs} 23 \mathrm{~min} 40.95 \mathrm{~s}$, and a declination of $10^{\circ} 21^{\prime} 48$. 2'. Since data smoothing techniques and filtering methods are fairly complicated subjects, a possible assignment can be derived using Ephemeris data. In this case, the classical Laplace or Gauss Methods use three observations of R.A. and Declination to determine the orbit. The mathematical formulas used are manageable in a classroom setting. Also, when using MATLAB programming techniques, results can be obtained with not too much programming efforts, and student satisfaction as proven by

\begin{tabular}{|c|}
\hline \multirow{2}{*}{2002 EL6 } \\
\hline \\
\hline K02E06L*C2002 $0310.13090142340 .95+102148.2$ \\
\hline K02E06L C200203 $10.13417142341 .24+102150.3$ \\
\hline K02E06L C2002 $0310.13729142341 .60+102152.9$ \\
\hline K02E06L C2002 $0310.14056142341 .86+102154.4$ \\
\hline K02E06L C2002 $0310.92956142457 .82+103036.1$ \\
\hline K02E06L C2002 $0310.93229142458 .10+103037.5$ \\
\hline
\end{tabular}


end of semester questionnaires is very high. (Figure2.4) depicts a student programmed graphical user interface with the calculated orbit, which is based on the observations.

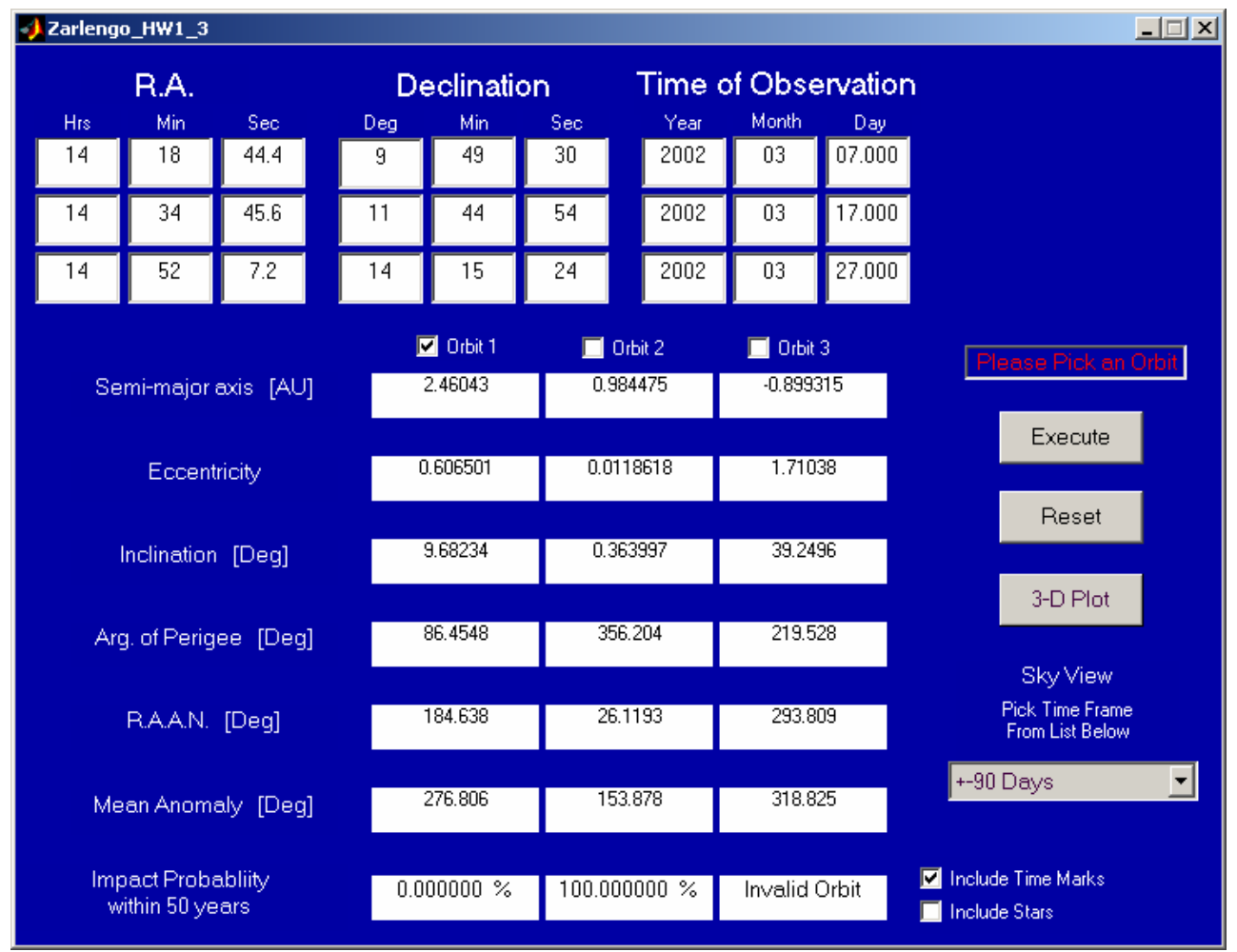

Figure 2.4

Since the Laplace method generally yields three solutions, where only one is the valid answer, the student needs to apply additional reasoning to find the proper result. The simplifications for this problem are substantial, but on the other hand, the student learns all the relevant tools for mastering this type of assignment.

\subsection{Fragmentation Catalog Project}

An interdisciplinary assignment was the generation of an electronic Fragmentation Catalog, similar to the one, which is maintained at NASA/JSC [4]. For this project the student performed an independent study, worth 3 credit hours. The educational value is in database design, graphical user interface design, and the general understanding of satellite fragmentations. This project also served as an instructional tool for orbit mechanics, satellite cataloging, and orbit evolution.

\subsection{Space Craft Design Projects}

The curriculum at Embry Riddle Aeronautical University requires a spacecraft preliminary design and spacecraft detail design sequence. The preliminary design is about feasibility studies, whereas the detail design is concerned with building "Space Hardware". Two of the three Fall 2003 Preliminary Design class teams chose to design satellite platforms to measure debris in the space environment $\left(70^{\circ}-110^{\circ}\right.$ inclination, $800-1100 \mathrm{~km}$ altitude $)$. One team utilized a PVDF 
(Polyvinylidene Fluoride Piezoelectronic Foil) based impact detector, while the other team employed (conceptually) a DEBIEtype impact sensor. Both teams integrated the sensor package (complete with on-board memory, data processing, telemetry \& housekeeping system design) with a simple micro satellite bus. This required the students to model all relevant systems, including structures, thermal, attitude determination and control, communications, etc. The

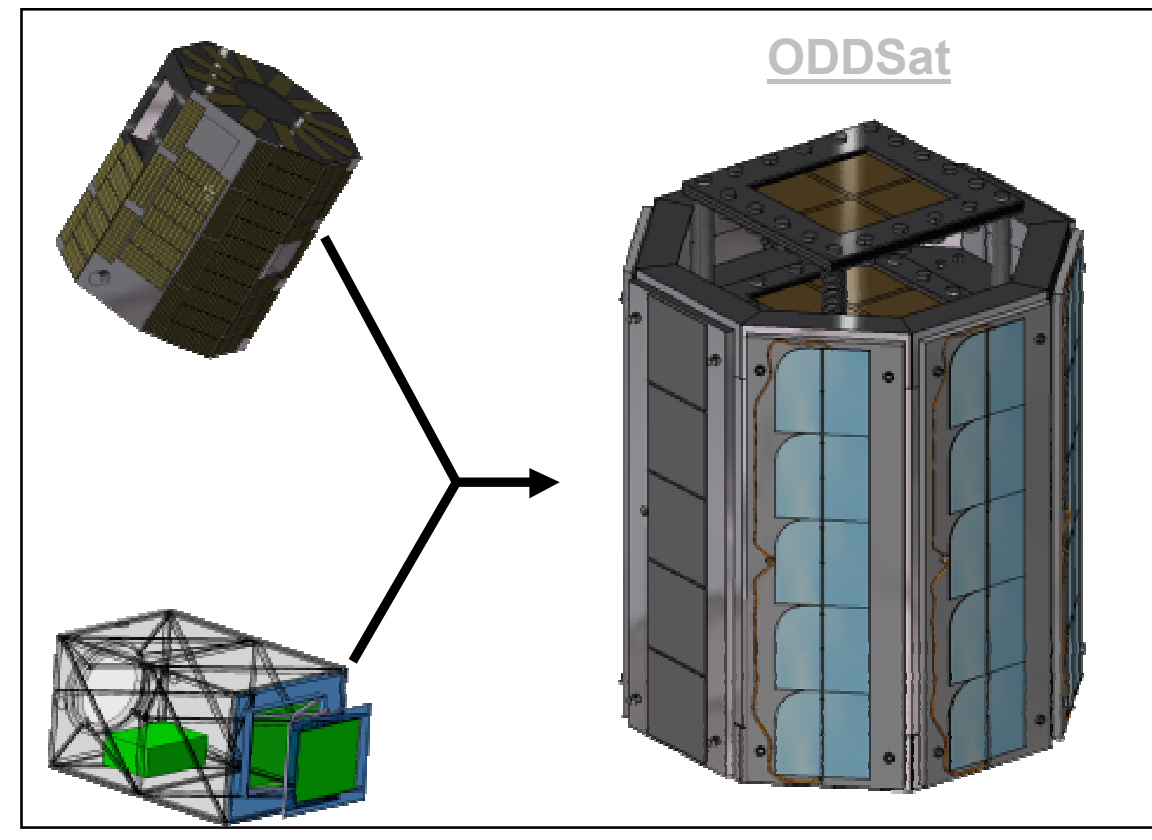

Figure 2.6

Spring ' 04 capstone design class condensed all three groups into one group, indicative of the increased work-load associated with hardware \& software engineering vs. "paper studies".

Salient features of all three team's preliminary designs, including the PVDF debris payload, were incorporated into the capstone product, the Orbital Debris Detector satellite (ODDSat) (Figure 2.6). Students tested the PVDF payload through actual impacts in a laboratory. The students used available NASA Engineering computer models to estimate projectile speed, size, and directionality.

One important feature of the university environment is the capacity to serve as a repository of knowledge and to develop this knowledge base. In order to take advantage of the design projects, the ERAU chapter of the Society of Women Engineers (SWE) under the auspices of the NASA Space Grant Program, is currently validating design concepts to plan, assemble, and fly a space craft whose mission is to measure the space debris environment down to $1-10 \mu \mathrm{m}$ impact sizes.

\subsection{The Plasma Drag Accelerator (PDA)}

Also sponsored by the NASA Space Grant program is the construction of a plasma-drag accelerator [5] for impact phenomenology and spacecraft protection/shielding studies. Our initial, two-fold goal is to (a) characterize plasma erosion of the projectiles and (b) modify/enhance the original designs' structure to allow a greater frequency of firing the accelerator. The design project has been entirely performed by students, with actual construction performed by students working under the tutelage of the University's machinist.

The PDA has been completed as of this writing and shipped to Baylor University's Center for Astrophysics, Space Physics and Engineering Research (CASPER) in Waco, TX, for testing in their vacuum chambers (we will also be jointly making use of their diagnostics, high speed cameras, etc.). This will involve a joint faculty/student team. 


\subsection{Atmospheric Decay Prediction}

Sponsored initially by the College of Arts \& Science, and now by the NASA Space Grant program, we're investigating the effects of transient solar phenomena, e.g. solar flares and coronal mass ejection (CMEs) on the Earth's magnetosphere and upper atmosphere by examining the decay of low Earth orbit spherical satellites. A student has led this effort, which incorporates a knowledge of the space environment, solar and atmospheric chemistry and physics, orbital mechanics (including perturbations), and computer modeling in the FORTRAN language. This work has led to the student's first professional presentation at the December 2004 meeting of the American Geophysics Society (AGU) and will produce a scientific paper (final report) to be submitted for publication in 2006.

\subsection{The CCD Debris Telescope (CDT)}

A group of ERAU faculty and students have acquired NASA's CDT, which has been excessed under the Stevenson-Wydler Technology Innovation Act. The initial operational capability (IOC) has been achieved in the summer of 2005. Erection of the CDT has been performed by a student led team of Aerospace Engineering and Space Physics students, with minimal supervision by faculty or consultation with prior operators. The team is also supported by the McNair scholarship program, which supports undergraduate research opportunities. University monies have been allocated to the construction of a roll-on/-off roof observatory building for the CDT and space/resources sufficient to establish the "control room" and enhance CDT hardware over time. Construction is expected to be finished in the 2006 calendar year. Operations will involve student researcher teams working in conjunction with the authors and other faculty.

\section{Space Debris Awareness}

It is clear that every student in space engineering and space physics should have an exposure to space debris related topics [2] in order to enhance their situational awareness of the space environment. Engineering students should be cognizant of both protection \& mitigation strategies employed by government and industry. Space physicists should appreciate the dynamic nature of the debris environment as an exemplar of space physical mechanisms, as well as limitation or constraints which may be placed on their payloads, e.g. contamination of optical observations by debris "streak". Experimental methods used in observing debris or recording impacts is directly relatable to remote sensing, impact mechanisms or planetary cratering. It has been demonstrated, in the authors experience, that the topics generate high interest and are very interdisciplinary. They are also very well suited to explain effects in other areas of space mechanics, space systems engineering, computer science, statistics, space policy, and space law.

\section{Conclusions}

Space debris studies are highly specialized i.e. they are not a "growth industry" However, space debris studies are ideal for space-related degree programs (engineering, space physics, global security and intelligence, etc.) because they are highly interdisciplinary by requiring cross-training in both science and engineering, they impart strong background in mathematics 
and statistics, and generate knowledge of what's required to operate in the harsh space environment

\section{References}

[1] Kessler, D.J., 1984. Derivation of the Collision Probability between orbiting objects: The lifetime of Jupiter's outer Moons. Icarus 48:39-48

[2] Anonymous, Orbital Debris, A Technical Assessment, National Research Council, National Academy Press, Washington, D.C. 1995

[3] Minor Planet Center: http://cfa-www.harvard.edu/iau/mpc.html

[4] Satellite Fragmentation Catalog:

http://orbitaldebris.jsc.nasa.gov/library/SatelliteFragHistory/13thEditionofBreakupBook.pdf

[5] Igenbergs,E., 1973 Magnetogasdynamic compression of a coaxial plasma accelerator flow for micrometeoroid simulation, Appl. Phys, Vol 44, No.5, May 1973 